\title{
Analysis of Off-Nuclear X-Ray Sources in Galaxy NGC 4945
}

\author{
Sarah M. Harrison \\ Office of Science, Science Undergraduate Laboratory Internship (SULI) \\ Massachusetts Institute of Technology \\ Stanford Linear Accelerator Center \\ Stanford, CA
}

August 24, 2006

Prepared in partial fulfillment of the requirements of the Office of Science, Department of Energy's Science Undergraduate Laboratory Internship under the direction of Grzegorz Madejski and Martin Mueller at the Kavli Institute for Particle Astrophysics and Cosmology, Stanford Linear Accelerator Center.

Participant:

Signature

Research Advisor:

Signature 


\section{TABLE OF CONTENTS}

Abstract

$\begin{array}{ll}\text { Introduction } & 1\end{array}$

$\begin{array}{ll}\text { Materials and Methods } & 3\end{array}$

$\begin{array}{lr}\text { Results } & 6\end{array}$

$\begin{array}{ll}\text { Discussion and Conclusions } & 7\end{array}$

$\begin{array}{lr}\text { Acknowledgments } & 10\end{array}$

$\begin{array}{ll}\text { References } & 10\end{array}$ 


\begin{abstract}
Analysis of Off-Nuclear X-Ray Sources in Galaxy NGC 4945. SARAH M. HARRISON (Massachusetts Institute of Technology, Cambridge, MA 02139) GRZEGORZ MADEJSKI AND MARTIN MUELLER (Kavli Institute for Particle Astrophysics and Cosmology, Stanford Linear Accelerator Center, Stanford, CA 94025)
\end{abstract}

Recently, X-ray astronomy has been used to investigate objects such as galaxies, clusters of galaxies, Active Galactic Nuclei (AGN), quasars, starburst superbubbles of hot gas, X-ray binary systems, stars, supernova remnants, and interstellar and intergalactic material. By studying the x-ray emission patterns of these objects, we can gain a greater understanding of their structure and evolution. We analyze X-ray emission from the galaxy NGC 4945 using data taken by the Chandra X-ray Observatory. The Chandra Interactive Analysis of Observations (CIAO) software package was used to extract and fit energy spectra and to extract light curves for the brightest off-nuclear sources in two different observations of NGC 4945 (January, 2000 and May, 2004). A majority of sources were closely fit by both absorbed power law and absorbed bremsstrahlung models, with a significantly poorer $\chi^{2} /$ dof for the absorbed blackbody model, and most sources had little variability. This indicates that the sources are accreting binary systems with either a neutron star or black hole as the compact object. The calculated luminosities were about $10^{38} \mathrm{erg} / \mathrm{s}$, which implies that the mass of the accreting object is close to 10 solar masses and must be a black hole. 


\section{INTRODUCTION}

The study of X-ray sources provides information about the structure of the objects which make up the universe. Recently, X-ray data has been used to investigate objects such as galaxies, clusters of galaxies, Active Galactic Nuclei (AGN), quasars, starburst superbubbles of hot gas, X-ray binary systems, stars, supernova remnants, and interstellar and intergalactic material. By studying the X-ray emission patterns of these objects, we can gain a greater understanding of their structure and evolution. The process of accretion (the extraction of gravitational potential energy from a material which falls onto a gravitating body) is known to be the principal source of power in several types of binary systems and is also believed to power AGN and quasars [1]. The study of luminous x-ray sources can provide further insight into the accretion process in stellar systems.

There are three main mechanisms of astronomical X-ray production: thermal bremsstrahlung in an ionized gas, synchrotron radiation, and blackbody radiation. The acceleration of charged particles during collisions within a hot, ionized gas causes emission of radiation known as thermal bremsstrahlung. This radiation forms an energy continuum with a characteristic shape dependent on the temperature of the gas. It also may have characteristic emission lines based on the elemental composition of the gas. This type of radiation is often emitted from hot gas in clusters of galaxies and supernova remnants. Synchrotron radiation, also known as magnetic bremsstrahlung, is generated when a relativistic electron is accelerated by a magnetic field. An example of a synchrotron emitter is the central region of the Crab Nebula, a young supernova remnant in our galaxy, which has a very strong magnetic

field ( $10^{-4}$ gauss) [2]. X-rays are also produced by blackbody radiation of normal stars over a broad temperature range $(2,500 \mathrm{~K}$ to $40,000 \mathrm{~K})[2]$ and high-temperature neutron stars, among other objects. By measuring the x-ray spectrum and the shape of the continuum by fitting it with one of these models, the source emission can be classified as one of these types.

Measuring the luminosity of the sources can also give a lower limit for the mass of the 
objects, and possibly lead to the discovery of extremely luminous or massive off-nuclear sources. For many accreting astrophysical systems, there is a maximum luminosity which the system reaches. As material accretes onto a compact object, the gravitational potential energy lost is released in the form of electromagnetic radiation. This radiation exerts a force on the surrounding material, mostly on free electrons through Thompson scattering. At a point known as the Eddington limit, the outward force from the radiation balances the inward gravitational force, preventing any further increase in the rate of accretion. By balancing the radiative and gravitational forces, the mass of systems radiating at their Eddington luminosity can be determined[1].

Seyfert galaxies are spiral or irregular galaxies containing a strong nucleur source, most likely a supermassive black hole. Seyferts are a type of active galaxy, galaxies which emit a large fraction of their energy through a bright, compact, extremely massive galactic center. NGC 4945 is a nearby (3.7 Mpc)[3] Seyfert 2 galaxy known to have a very luminous AGN. The behavior of this nuclear region is currently the subject of detailed study. The spectra of the nucleus, starburst, and superwind regions were isolated in order to study the current model of black hole and accretion disk in Seyfert 2 galaxies[4]. In addition, a powerful offnuclear source has been observed in NGC 4945 which is thought to be an ultra-powerful x-ray binary or a supernova remnant[3].

In this paper, a number of off-nuclear sources in NGC 4945 will be investigated and catalogued using x-ray data taken by the Chandra X-ray Observatory (CXO). The classification of these sources will increase the depth of knowledge about X-ray binaries and other emitters and may lead to the discovery of new and interesting X-ray sources such as massive off-nuclear black holes. The discovery and analysis of ultraluminous sources, sources with luminosities greater than $2 * 10^{39} \mathrm{erg} / \mathrm{s}[5]$, can provide more information about the physics of intermediate mass black holes, black holes with masses between 10 and 100 solar masses. Only a few of these type of sources are known, and little is understood about their evolution. Other possible sources of these X-rays are supernova remnants, normal stars, and 
binary systems involving accreting white dwarf stars, neutron stars, or stellar black holes.

\section{MATERIALS AND METHODS}

\section{The Chandra Satellite}

The Chandra X-ray Observatory is composed of a high-resolution X-ray telescope and a number of advanced imaging and spectroscopic instruments. Launched on July 9, 1999, the Chandra satellite achieved the most advanced spatial and X-ray spectral resolution to date. The satellite is comprised of the Pointing Control and Aspect Determination (PCAD) system, which controls the pointing of the observatory, the High Resolution Mirror Assembly (HRMA), which focuses the X-rays, and the Science Instrument Module (SIM), which houses the two focal instruments, the High Resolution Camera (HRC) and the Advanced CCD Imaging Spectrometer (ACIS). In addition are the Low Energy Transmission Grating (LETG) and High Energy Transmission Grating (HETG) for use with the HRC and ACIS, respectively.

ACIS, the camera used for the observations analyzed in this paper, is composed of 10 planar 1024-by-1024 pixel charge coupled devices (CCDs) arranged in two arrays. One is a four-chip array called ACIS-I and the other is a six-chip array called ACIS-S. ACIS can obtain high-resolution images and moderate resolution spectra, but the use of ACIS-S in conjunction with the HETG can obtain very high resolution spectra. The HETG is composed of 2 sets

of gratings: the medium energy grating (MEG) and the high energy grating (HEG). Overall, the HETG is designed for high resolution spectroscopy between $0.4 \mathrm{keV}$ and $10.0 \mathrm{keV}[6]$.

\section{Data Acquisition and Analysis}

X-ray sources in the nearby galaxy NGC 4945 were analyzed and compared using data taken by the Chandra satellite during two observations, one in 2000 and one in 2004 . The first observation began on January 27, 2000 (total exposure time $49.75 \mathrm{ks}$ ) with the ACIS camera 
in faint mode and did not use a grating. The second observation began on May 28, 2004 and was split into two data sets (total exposure times $78.64 \mathrm{ks}$ and $95.8 \mathrm{ks}$, with $57.6 \mathrm{ks}$ gap). Both observations were taken with the ACIS camera in faint mode and with a high energy transmission grating (HETG) placed in front of the camera. The data was retrieved from the Chandra archive and analyzed using the Chandra Interactive Analysis of Observations (CIAO) software package[7]. Two main files for each observation were used in data analysis. The first is a source file, a table of all the sources detected, their locations in the sky, their net count rate and background counts, along with other information. The second is an event file, which allows the extraction of an image of net photon events at each pixel position, like the one shown in figure 1 . The earlier observation had to be reprocessed because many of the software threads had been updated since the original processing in 2000. This included the removal of afterglow effects caused by residual charge left by the interaction of cosmic rays with the CCD. This excess charge is captured by charge traps and persists through several subsequent frames of CCD read-out and could result in spurious detection of faint sources. Certain pixels which may have been illuminated by extra charge were recorded in an updated file in order to remove them from data analysis. The event and source files provided for the 2004 observations had already been processed with the most recent techniques in CIAO and did not need to be reprocessed.

The brightest X-ray sources in each of the observations were determined using source files provided by the Chandra archive. The images of NGC 4945 were displayed with the event file. This image was used to locate and extract circular regions (of radius 5 pixels) around each of the brightest sources in order to extract energy spectra for these sources. In addition, a source-free background region for each observation was used to subtract a background count from the source regions when producing spectra. A tool from the CIAO package was used to extract these spectra as well as the Auxilary Response File (ARF) and Redistribution Matrix File (RMF) for each source. These files are used during spectral fitting to take into account the effective detector area and detector ineffeciencies in energy 
resolution, as well as the transition from incoming photon energy to the value recorded by the detector. These spectra display the photon energy versus the number of photons in each energy range and can be used to determine the nature of the X-ray emission. The spectral fitting technique used assumes a model with reasonable parameters, propogates it through the instrument by taking into account the $\mathrm{ARF}$ and $\mathrm{RMF}$, and compares this output to the data. Then the parameters are adjusted until a minimum $\chi^{2}$ value is reached.

Three different fit models were applied to each source spectrum using the program xspec. Absorbed power law, absorbed bremsstrahlung, and absorbed blackbody models were compared to each other for each of the sources, and model parameter values and $\chi^{2} /$ dof were calculated for each. For each model, the flux was used to calculate the luminosity of the source by the formula:

$$
L=4 \pi r^{2} f
$$

where $f=$ the flux of the source and $r$ is the distance to the galaxy (3.7 Mpc).

This calculated luminosity was used to determine a lower limit for the mass of each source and to indicate whether a black hole or a neutron star was present for those sources which were accreting x-ray binary systems.

Light curves, plots of the change in net photon count rate with time over the course of the observation, were extracted and plotted for these sources using CIAO. In each case, the plots were binned in $3000 \mathrm{~s}$ intervals. In order to take into account the grating used in observations 4899 and 4900, the curves were rescaled using the count rates calculated for the nuclear source in each of the observations which is known not to be variable. The light curves were used to study the emission rate of each source and to assess its variability. Both the light curves and energy spectra for each observation were compared for each source in order to study its time evolution. In addition to the brightest sources, sources which were present in one observation and not detectable in another were analyzed. A catalog of the brightest sources in the galaxy NGC 4945 was produced. 


\section{RESULTS}

Table 1 displays spectral fitting information for the five sources with the greatest count rate (listed in order) in each observation. These sources are labeled in order of decreasing count rate in observation 864 and keep this same number across observations. The position of each source is provided in units of right ascention and declination, and the count rate is measured within the energy range $0.5-10 \mathrm{keV}$ (the range used for fitting). The spectral parameters for each of the three fits used are displayed, including $n_{H}$, the absorption column density for the absorbed component of each model, the photon index for the power law model, and the temperature for the bremsstrahlung and blackbody models. The $\chi^{2} /$ dof value calculated for each model is displayed, along with the flux calculated for each fit (in the energy range 2-10 $\mathrm{keV}$ ) and the luminosity of the source. In observation 864, the four brightest sources were best fit by absorbed thermal bremsstrahlung models, and the fifth brightest source was best fit by an absorbed power law model. In each case, the $\chi^{2} /$ dof values for absorbed power law or bremsstrahlung were fairly close to each other and significantly better than that for the absorbed blackbody model. The luminosities for these sources ranged from $9 * 10^{38} \mathrm{erg} / \mathrm{s}$ for the brightest source to $2 * 10^{38} \mathrm{erg} / \mathrm{s}$ for the fifth brightest source.

Except for the fifth brightest source (source 6) in observation 4899, where each model had a $\chi^{2} /$ dof of 1.5 , the $\chi^{2} /$ dof values for absorbed power law and bremsstrahlung models remained similar and significantly lower than that for the absorbed blackbody model. Sources 3, 7, and 5 in observation 4900 also show this characteristic.

The spectral parameters for sources 1 through 7 in the observations in which they were not one of the five brightest sources are displayed in table 2 . Source 7 was not bright enough to be detected in observation 864. Two moderately bright sources were observed in observation 864 but were not noticeable in observations 4899 and 4900. The spectral parameters for these sources are displayed in table 3.

Figures 2 through 10 display the lightcurves for sources 1 through 9 in each of the 
observations in which they were noticed. Most sources, except for source 7 which shows high variability, show little or no variability over the course of each observation.

\section{DISCUSSION AND CONCLUSIONS}

Most sources were best fit with absorbed power law and absorbed bremsstrahlung models, which have similar shape. The fit of absorbed power law indicates that these sources are accreting x-ray binaries, with either a neutron star or black hole as the compact object. The luminosity range of $2-9 * 10^{38} \mathrm{erg} / \mathrm{s}$ indicates masses of a few solar masses, stars which are most likely black holes. The hydrogen column density calculated for absorption for most

models is $2-7 * 10^{21} \mathrm{~cm}^{-2}$, which is smaller than the $n_{H}$ calculated by Done to be $4 * 10^{24}$ $\mathrm{cm}^{-2}$ and closer to the reported absorption of our own galaxy, which is $2 * 10^{21} \mathrm{~cm}^{-2}[4]$.

Because observations 4899 and 4900 were taken with the high energy transmission grating in front of the ACIS camera, fewer photon events were recorded for each source than in observation 864, and the overall count rates were smaller. Statistically, the fits to the data from observation 864 were more accurate because they incorporated more events. The sparsity of counts in the later observations may have caused the fitting to be less accurate, especially with the fainter sources, where the number of pha bins used for fitting was less than 25, whereas the faintest source in 864 had 50 pha bins. In addition the light curves for observation 864 reflect the variability of the sources more accurately because more counts were included in each bin.

Source 1: This source is one of the five most luminous sources in each observation. It was consistently fit reasonably well by both the absorbed power law and absorbed bremsstrahlung models. The count rate decreased by an order of magnitude between observation 864 (0.059 cts/s) and observations $4899(0.0064 \mathrm{cts} / \mathrm{s})$ and $4900(0.0060 \mathrm{cts} / \mathrm{s})$. The luminosity also decreased significantly between the observations, going from $9 * 10^{38} \mathrm{erg} / \mathrm{s}$ in 864 , a luminosity on the order of ultraluminous sources $\left(10^{39} \mathrm{erg} / \mathrm{s}\right)$ to 5 or $6 * 10^{38} \mathrm{erg} / \mathrm{s}$ in 4899 and 4900 . 
This suggests that the luminosity of this source is decreasing.

Source 2: In all three observations, this source was best fit by an absorbed bremsstrahlung model. The absorbed power law fit was also a close fit, with a photon index consistently between 2.3 and 2.5. The luminosity of this source also significantly decreased between observation $864\left(5.6 * 10^{38} \mathrm{erg} / \mathrm{s}\right)$ and observations 4899 and $4900\left(1.7 * 10^{38} \mathrm{erg} / \mathrm{s}\right)$, suggesting this source is getting fainter.

Source 3: This source, observed to be the third brightest in observation 864, was the most luminous in observations 4899 and 4900 . It was best fit by an absorbed bremsstrahlung model in all three observations, but was also closely fit by an absorbed power law model. For the power law model, photon index was consistently between 1.8 and 2.0. The luminosity increased from about $4 * 10^{38} \mathrm{erg} / \mathrm{s}$ in observation 864 to over $5 * 10^{38} \mathrm{erg} / \mathrm{s}$ in observations 4899 and 4900, indicating an increase in brightness of this source.

Source 4: The luminosity of this source remained consistent between all three observations, with a value between 2.5 and $2.9 * 10^{38} \mathrm{erg} / \mathrm{s}$ for the best fit models, which were absorbed bremsstrahlung and absorbed power law. This source does not show any variability.

Source 5: Like all of the other sources mentioned so far, this source was best fit by both absorbed power law and absorbed bremsstrahlung models. Its luminosity is consistent between the three observations, with a value between 1.7 and $2.1 * 10^{38} \mathrm{erg} / \mathrm{s}$. This source does not show any variability.

Source 6: This source, which had the fifth highest count rate in observation 4899, could not be well fit with any of the models for this observation. The $\chi^{2} /$ dof for each model was $>$ 1.5. In observations 864 and 4900, this source was best fit by an absorbed power law model with photon index of 1.5 and 2.5, respectively, and luminosities of $2.4 * 10^{38} \mathrm{erg} / \mathrm{s}$ and $3.3 * 10^{38} \mathrm{erg} / \mathrm{s}$, respectively. Because of the low count rate this source had in observation 4899 and 4900, it was more difficult to fit accurately.

Source 7: This source was too faint in observation 864 to extract an analysis, was observable in observation 4899, and was the third brightest source in observation 4900. In both 
observations 4899 and 4900, it was best fit by an absorbed power law model. Its luminosity was observed to increase from $2.0 * 10^{38} \mathrm{erg} / \mathrm{s}(4899)$ to $4.4 * 10^{38} \mathrm{erg} / \mathrm{s}(4900)$ over the short span between the two observations. The fact that it was barely observable in observation 864 indicates that it may have been flaring during the time of observations 4899 and 4900 .

Source 8: The absorbed bremsstrahlung model provided the best fit for this source, and gave a calculated luminosity of $1.3 * 10^{38} \mathrm{erg} / \mathrm{s}$. The absorbed power law model also provided a relatively good fit, with photon index of 2.4 and a similar calculated luminosity of $1.5 * 10^{38} \mathrm{erg} / \mathrm{s}$. This source, one of the fainter ones in observation 864, was not observable in observations 4899 and 4900, indicating a variable luminosity.

Source 9: This source is the only one which was best fit with the absorbed blackbody model. It was also reasonably well fit by the absorbed bremsstrahlung model. The faintest source described here, this source had a luminosity between 1.1 and $1.3 * 10^{37} \mathrm{erg} / \mathrm{s}$ during observation 864 and was not noticeable in observations 4899 and 4900, indicating a variable luminosity.

With luminosities in the range $<10^{39} \mathrm{erg} / \mathrm{s}$, it is unlikely that these sources are ultraluminous X-ray sources. The luminosities of these sources are in the same range $\left(10^{38} \mathrm{erg} / \mathrm{s}\right)$ as the luminosities of about 5 of the black hole candidates in our own galaxy as reported in Tanaka and Lewin[8]. The ratio of the luminosities of the sources in NGC 4945 to the Eddington luminosity for a source of one solar mass is a little under 10, and because it is unlikely that the sources are radiating at their Eddington limit (therefore this ratio underestimates the mass), these sources must be accreting black holes of almost 10 solar masses. The evidence of slight variability in the light curves rules out the possibility that most of these sources are supernovae, which would have very stable light curves without any variability. It can be concluded that NGC 4945 has more off-axis black holes than our own galaxy, which are in accreting binary systems and are emitting X-rays at luminosities close to the threshhold for ultraluminous X-ray sources.

A comparison with the analysis by Done et al.[4] on the nuclear source in NGC 4945 
verifies the analysis techniques used in this paper. Table 5 displays a comparison of the spectral parameters found by modeling the nuclear source with an absorbed powerlaw + gauss model in all three observations with the parameters for the model obtained by [4]. In each observation the presence of the FeK $\alpha$ line closely matches the $6.4 \mathrm{keV}$ value found by Done et al. The photon index is not similar between the two separate analyses, but this may have to do with the difference in the definition of the extraction region between the two different methods.

Further study of the variability of the sources could further confirm the conclusion that the brightest sources reported here are indeed black hole X-ray binaries. In addition, it could indicate more about the astrophysical process powering these X-ray sources which is most likely accretion. A search for optical sources at the same coordinates in observations taken by the Hubble telescope could provide more accurate information about the luminosities of these sources, as well as their activity in the optical band.

\section{ACKNOWLEDGMENTS}

I would like to thank my mentor, Dr. Grzegorz Madejski, and Martin Mueller for giving me guidance in this project. I would also like to thank Adam Edwards and all who read and aided me in writing this paper. Finally, I would like to thank the Department of Energy for sponsoring the SULI program and supporting youth in scientific research.

\section{REFERENCES}

[1] J. Frank et al., Accretion Power in Astrophysics, 2nd ed. Cambridge University Press, 1992.

[2] P. A. Charles and F. D. Seward, Exploring the X-Ray Universe. Cambridge University Press, 1995. 
[3] W. N. Brandt et al., "A powerful and highly variable off-nuclear x-ray source in the composite starburst/seyfert 2 galaxy ngc 4945," Mon. Not. R. Astron. Soc., vol. 281, pp. L41-L45, 1996.

[4] C. Done et al., "Simultaneous chandra and rossi x-ray timing explorer observations of the nearby bright seyfert 2 galaxy ngc 4945," The Astrophysical Journal, vol. 558, pp. $763-770,2003$.

[5] H. Feng and P. Kaaret, "Xmm-newton observations of ultraluminous x-ray sources in nearby galaxies," The Astrophysical Journal, vol. 633, pp. 1052-1063, 2005.

[6] The Chandra Proposers' Observatory Guide, 2nd ed. Chandra X-Ray Center, Chandra Project Science MSFC, and Chandra IPI Teams, 2000.

[7] "Chandra x-ray observatory," 2006. [Online]. Available: http://chandra.harvard.edu

[8] Y. Tanaka and W. H. G. Lewin, "Black-hole binaries," X-Ray Binaries, 1995. 


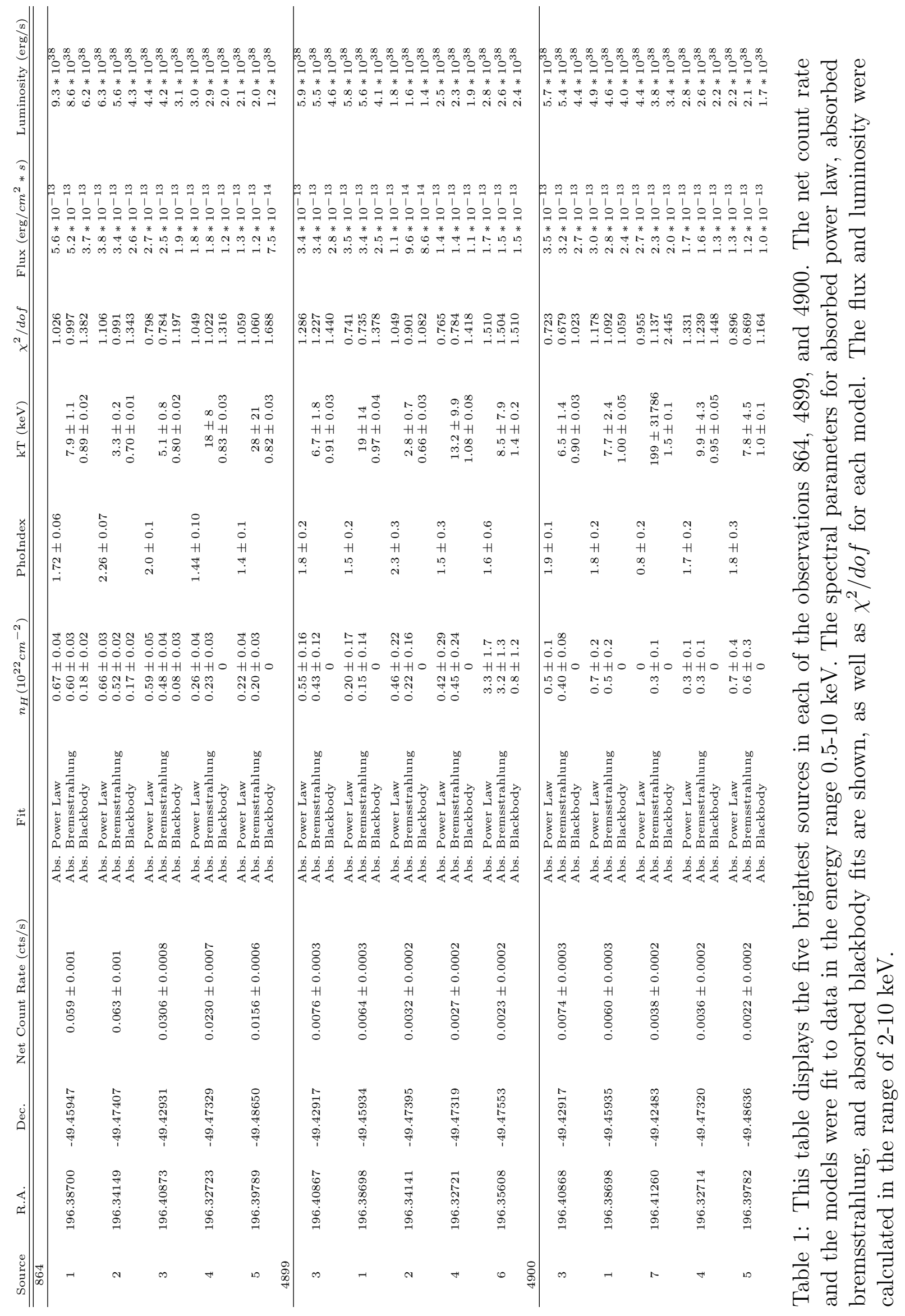




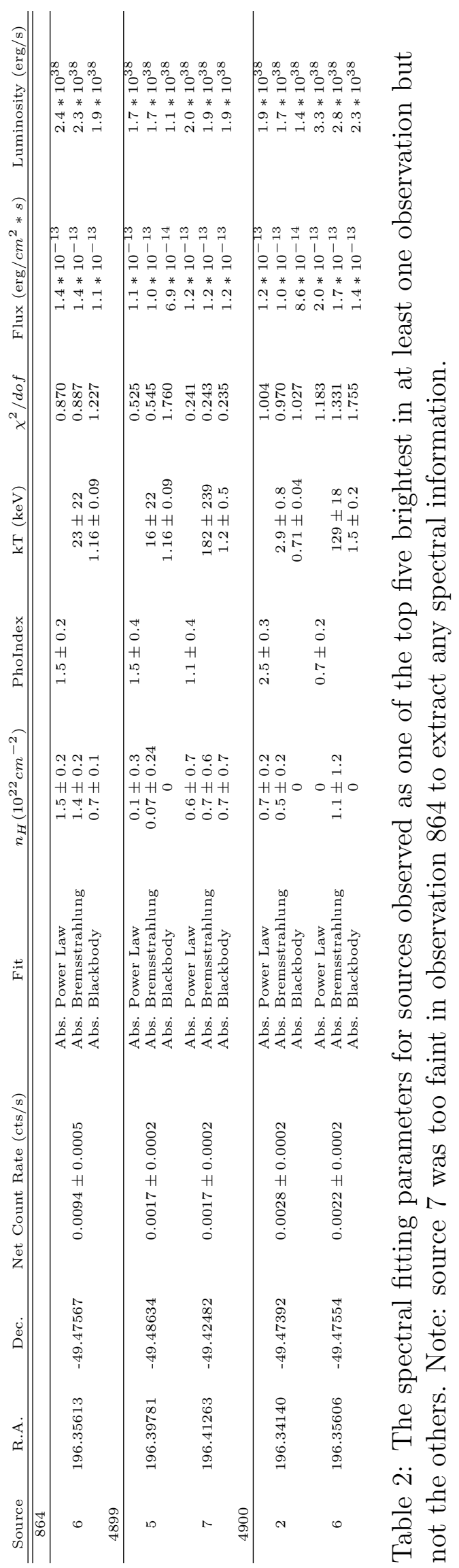




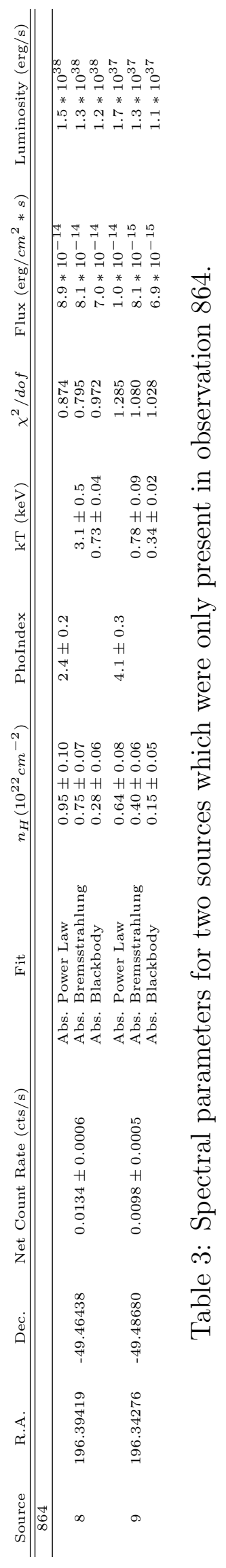




\section{FIGURES}

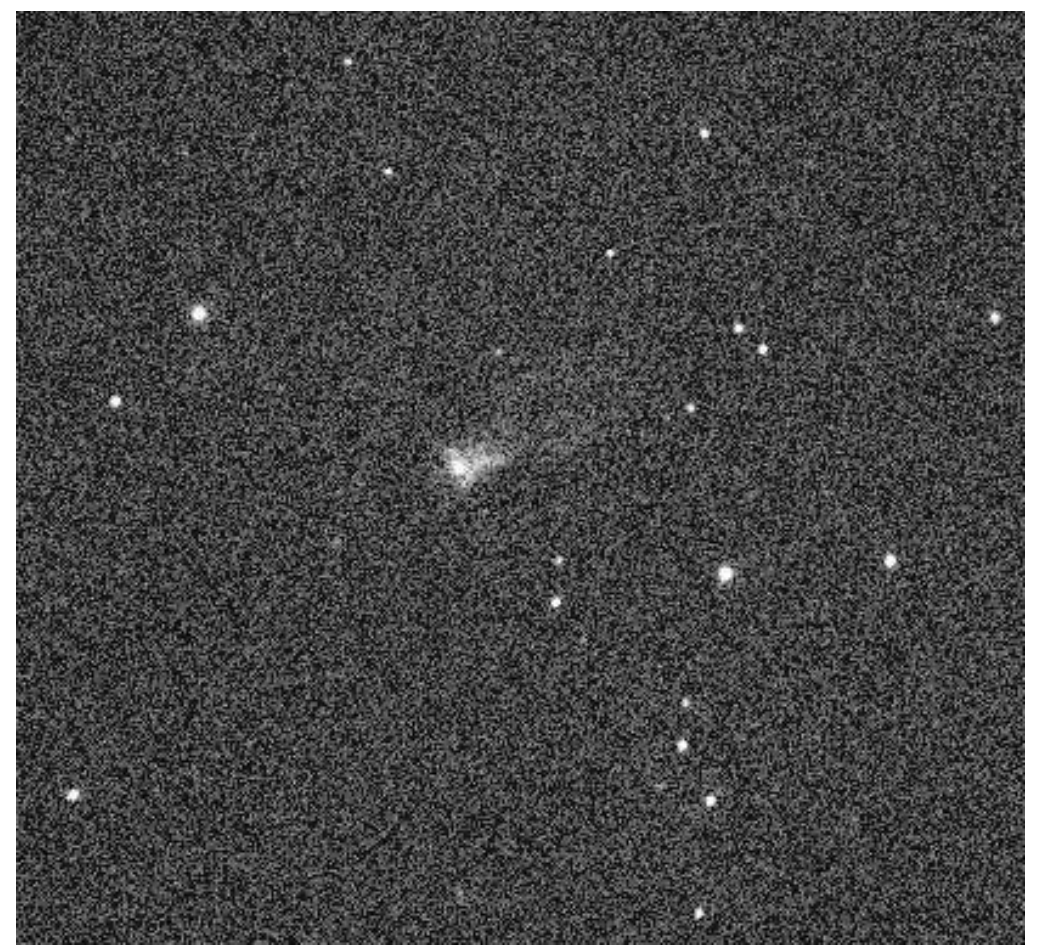

Figure 1: Chandra ACIS image of NGC 4945 from observation 864. 

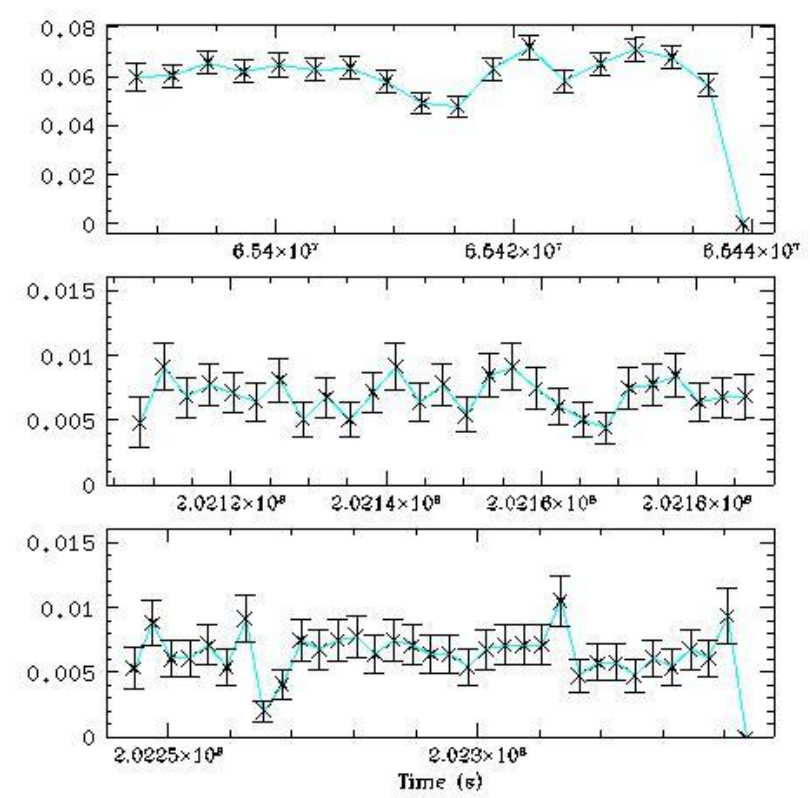

Figure 2: Source 1 light curves for observations 864, 4899, and 4900.

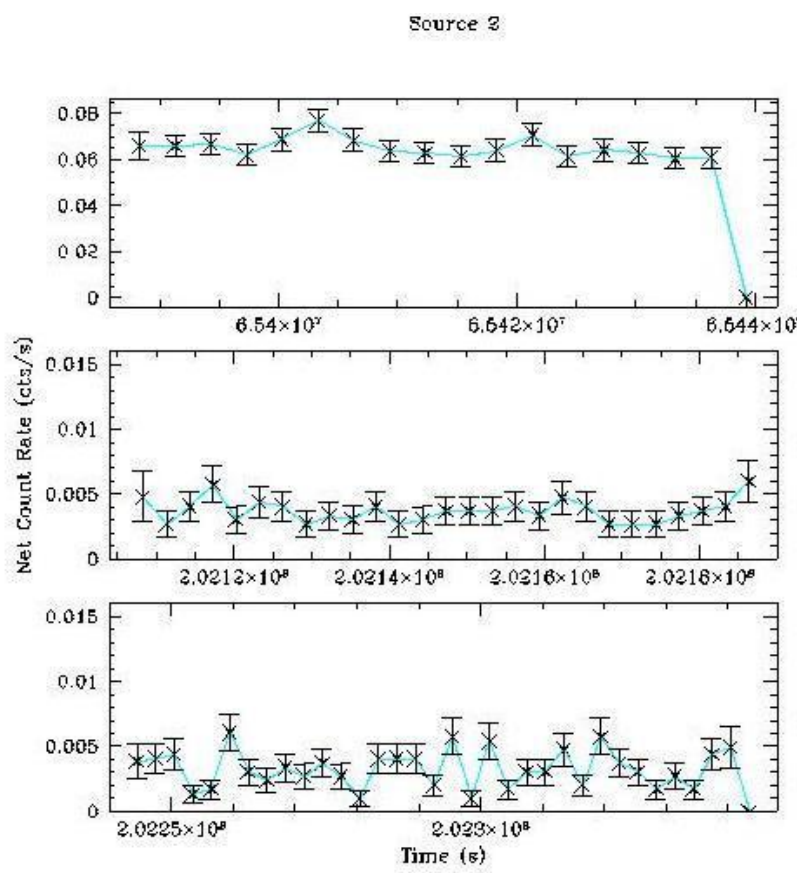

Figure 3: Source 2 light curves for observations 864, 4899, and 4900. 
Source 3
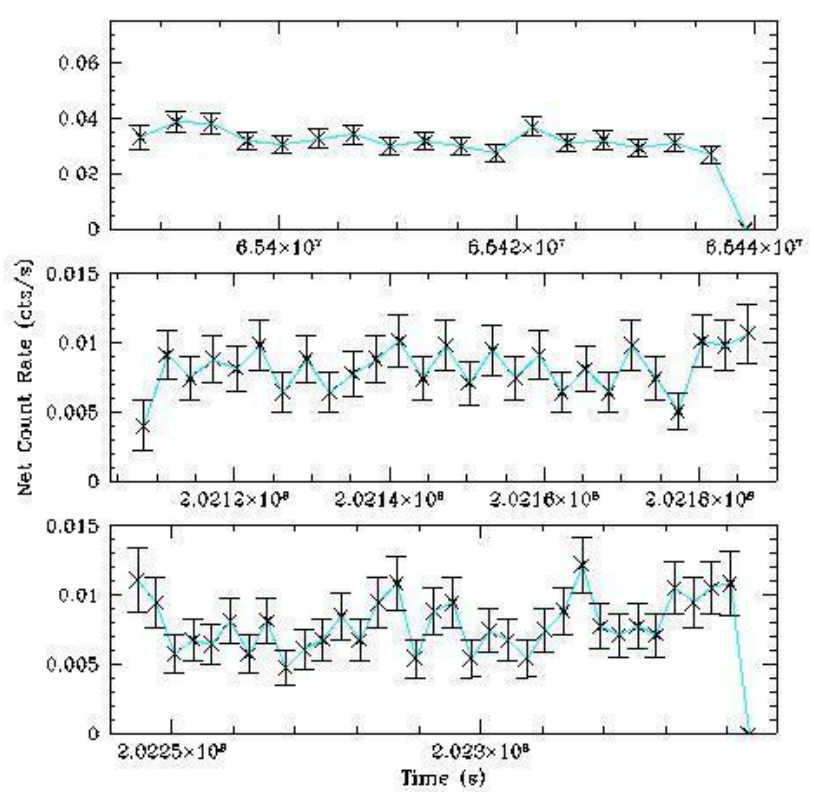

Figure 4: Source 3 light curves for observations 864, 4899, and 4900.

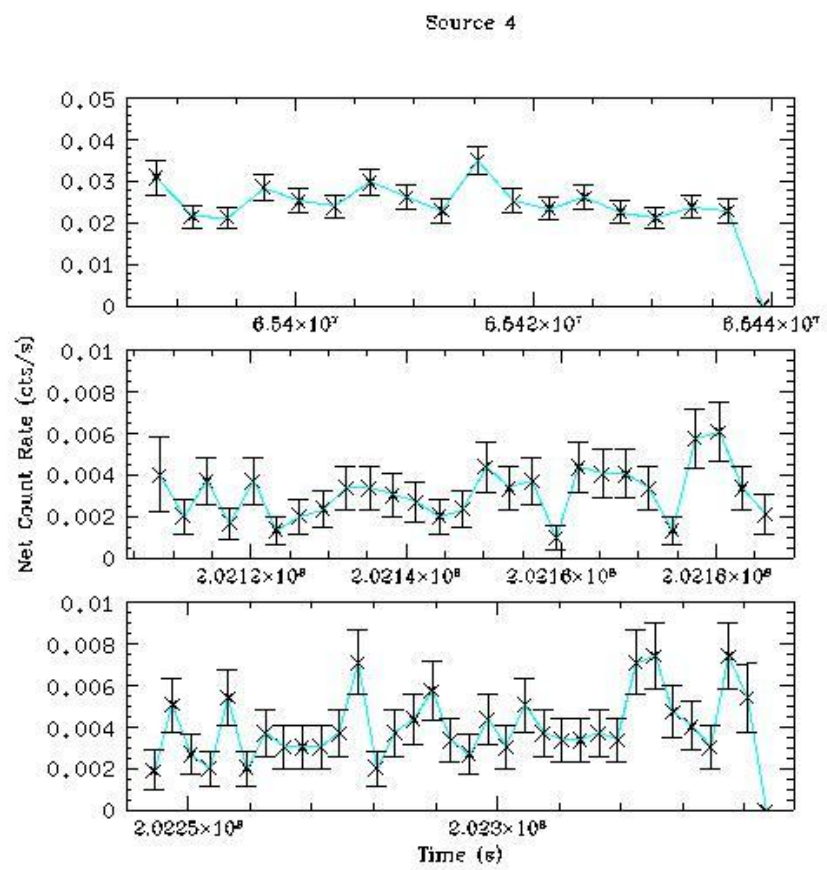

Figure 5: Source 4 light curves for observations 864, 4899, and 4900. 


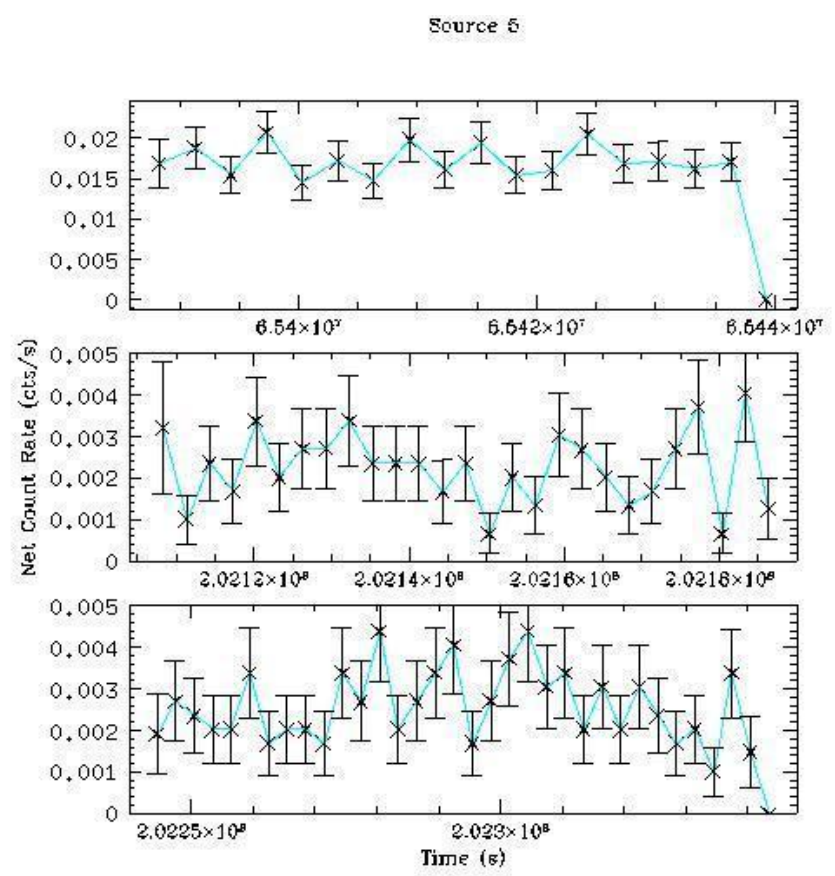

Figure 6: Source 5 light curves for observations 864, 4899, and 4900.

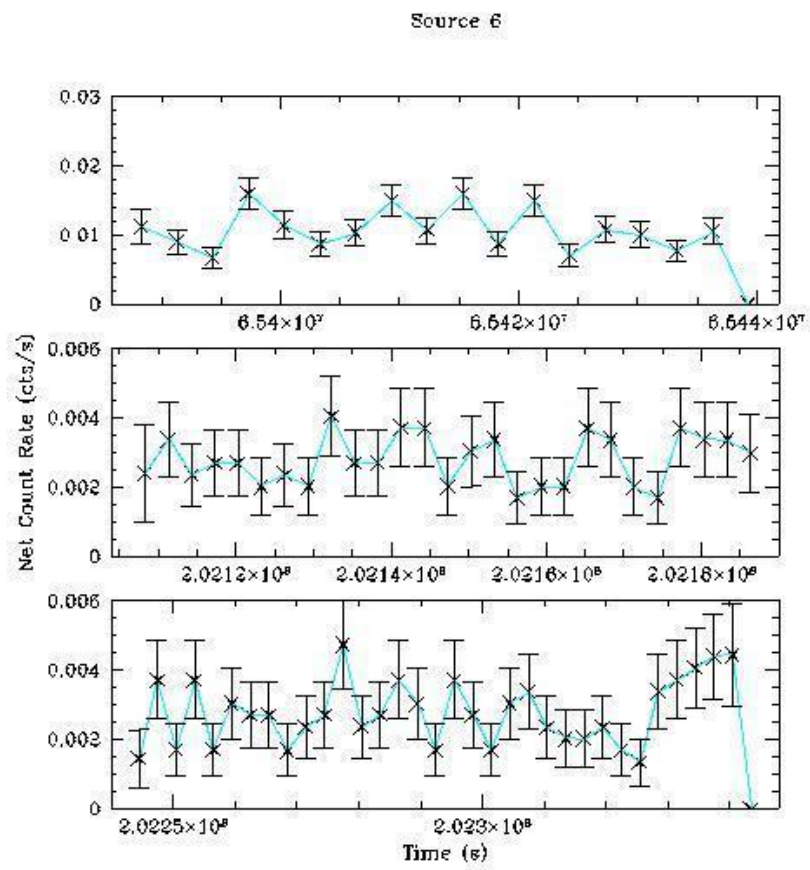

Figure 7: Source 6 light curves for observations 864, 4899, and 4900. 

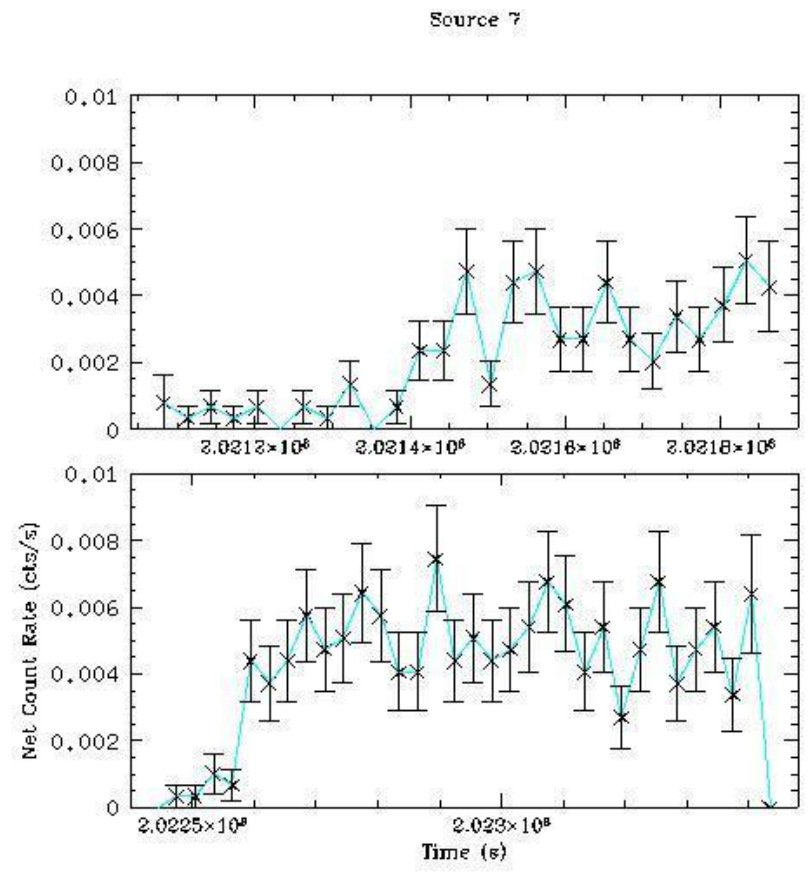

Figure 8: Source 7 light curves for observations 4899 and 4900.

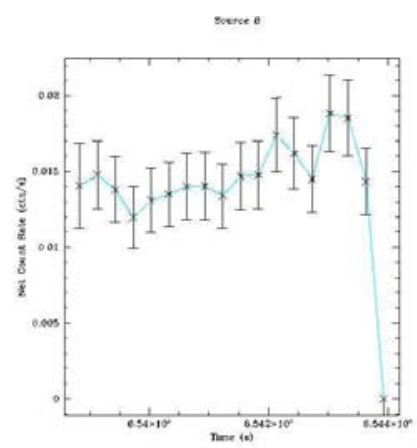

Figure 9: Source 8 light curve for observation 864. 


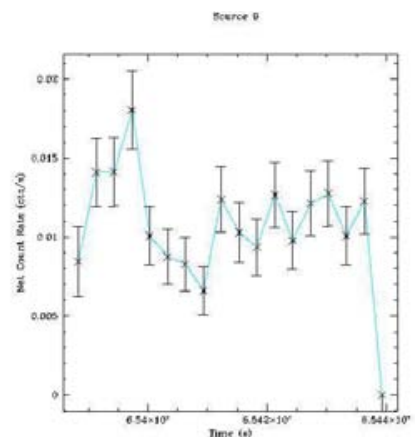

Figure 10: Source 9 light curve for observation 864 . 


\section{TABLES}
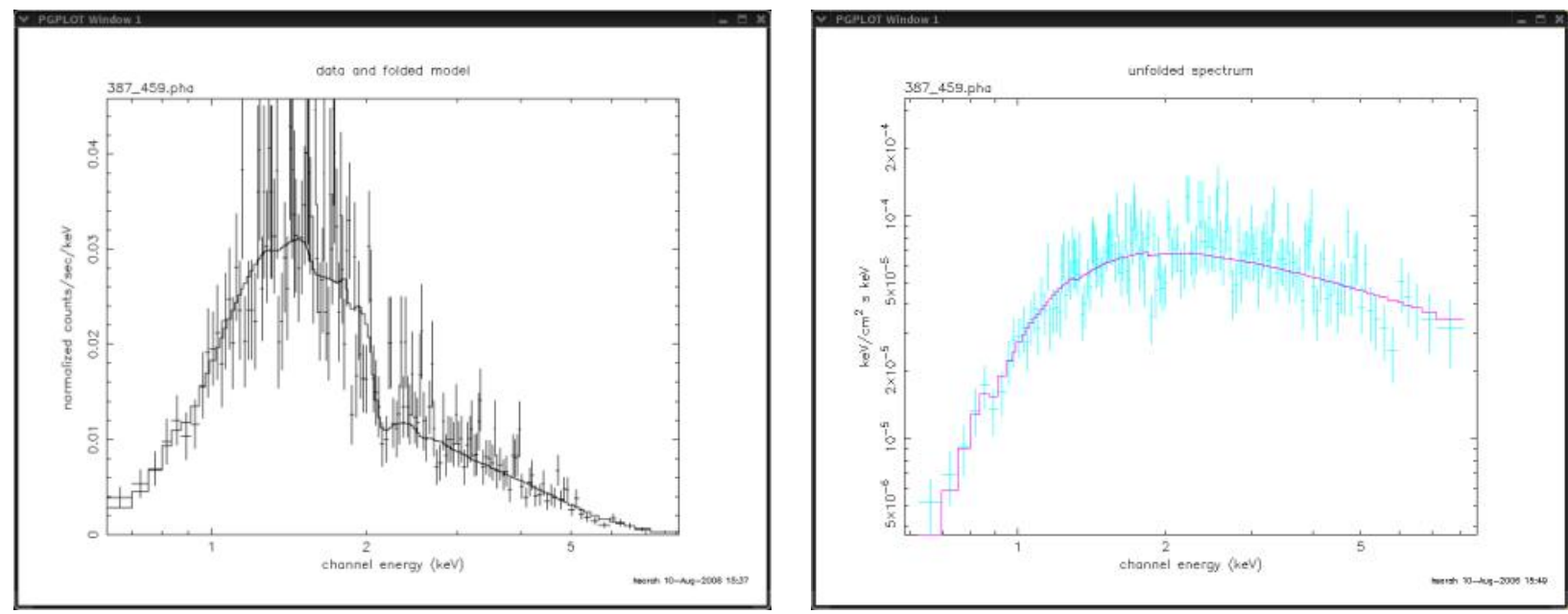

Table 4: Folded spectrum (left) and unfolded spectrum (right) for source 1 in observation 864, fitted with power law model.

\begin{tabular}{c|c|c|c||c} 
& 864 & 4899 & 4900 & Done et al. \\
\hline \hline$n_{H}\left(10^{22} \mathrm{~cm}^{-2}\right)$ & $1.1 \pm 0.2$ & $1.4 \pm 0.5$ & $1.4 \pm 0.5$ & $0.8 \pm 0.8$ \\
PhoIndex & $0.03 \pm 0.1$ & $-0.1 \pm 0.2$ & $-0.03 \pm 0.2$ & $1.65 \pm 0.15$ \\
FeK $\alpha(\mathrm{keV})$ & $6.4 \pm 0.2$ & $6.41 \pm 0.01$ & $6.385 \pm 0.007$ & 6.4 \\
$\chi^{2} /$ dof & 1.61 & 1.23 & 1.69 & 0.95
\end{tabular}

Table 5: Comparison of spectral parameters for fitting of nuclear source with absorbed powerlaw + gauss model between observations 864, 4899, and 4900 with those obtained by Done et al.. 